TTR

Traduction, terminologie, re?daction

\title{
De la co-présence pacifique à la co-présence créatrice : Le kaléidoscope des langues dans la littérature judéo-maghrébine
}

\section{Colette Touitou-Benitah}

Volume 9, numéro 1, 1er semestre 1996

Le festin de Babel

Babel's Feast

URI : https://id.erudit.org/iderudit/037241ar

DOI : https://doi.org/10.7202/037241ar

Aller au sommaire du numéro

Éditeur(s)

Association canadienne de traductologie

ISSN

0835-8443 (imprimé)

1708-2188 (numérique)

Découvrir la revue

Citer cet article

Touitou-Benitah, C. (1996). De la co-présence pacifique à la co-présence créatrice : Le kaléidoscope des langues dans la littérature judéo-maghrébine. TTR, 9(1), 117-137. https://doi.org/10.7202/037241ar
Résumé de l'article

De la co-présence pacifique à la co-présence créatrice : le kaléidoscope des langues dans la littérature judéo-maghrébine - Considérant la littérature judéo-maghrébine d'expression française depuis sa naissance en 1896 et jusqu'à nos jours sous l'angle de la co-présence de langues différentes dans le texte, trois périodes distinctes sont relevées. La première - de 1896 au début des années 50 - et la troisième - de 1962 à nos jours — sont marquées par cette co-présence. Mais cette co-présence a un caractère différent au cours de ces deux périodes. De la co-présence pacifique où le texte français était émaillé de mots et expressions en langues juives - judéo-arabe et judéo-espagnol on passe à une co-présence créatrice où les langues se fécondent l'une l'autre. Tous les jeux sont permis pour restituer ces langues juives qui se perdent et que l'on ne peut plus transmettre. La période intermédiaire au cours des années 50 constitue une rupture puisque des textes monolingues attestent du triomphe de la colonisation linguistique.
Tous droits réservés (C TTR: traduction, terminologie, rédaction — Les auteurs, 1996
Ce document est protégé par la loi sur le droit d'auteur. L'utilisation des services d'Érudit (y compris la reproduction) est assujettie à sa politique d'utilisation que vous pouvez consulter en ligne.

https://apropos.erudit.org/fr/usagers/politique-dutilisation/ 


\section{De la co-présence pacifique à la co-présence créatrice : Le kaléidoscope des langues dans la littérature judéo-maghrébine}

\section{Colette Touitou-Benitah}

\section{Introduction}

Avec l'arrivée des expulsés d'Espagne au $\mathrm{XV}^{\mathbb{e}}$ siècle, la culture juive au Maghreb a eu quatre " possibilités " linguistiques : l'hébreu, langue sacrée; le judéo-arabe et/ou le judéo-espagnol langues vernaculaires; l'arabe, langue dominante. En outre, du XV au XIX ${ }^{e}$ siècles en Tunisie et en Algérie, le turc fut la langue de domination.

M. Bar Asher (1992), J. Chetrit (1980, 1994) et J. Tedghi (1995) ont montré dans leurs travaux consacrés aux diverses langues de communication et aux textes de la littérature traditionnelle des Juifs du Maghreb que la co-présence de langues et la multiplicité des emprunts - attestées dèjà dans la littérature talmudique et rabbinique - était un modèle récurrent qui ne reflétait aucune tension sociale ou culturelle.

La colonisation française dans les trois pays du Maghreb donnera naissance à un courant de littérature judéo-maghrébine d'expression française dont la spécificité ne fait l'objet de recherches que depuis deux décennies. G. Dugas (1990) a montré l'originalité formelle et thématique de ce courant par rapport aux deux autres 
courants littéraires maghrébins qui lui sont contemporains : celui des Français de la colonie et celui des arabo-musulmans, mais ne s'est pas intéressé à la persistance du métissage des langues chez ses représentants.

Il a cependant noté très clairement les réactions opposées des deux groupes indigènes face à la langue du colonisateur:

Déculturation et contrainte pour les uns, acculturation vécue comme un enrichissement pour les autres : de cette énorme différence de perception découle, pour une bonne part, l'attitude fondamentalement divergente des intellectuels maghrébins et judéo-maghrébins face à la langue et à la culture françaises. (Dugas, 1990, p. 34)

Cette " différence de perception " a des racines socio-historiques qu'il n'est pas inutile de rappeler. Les Juifs furent dans leur ensemble naturalisés français en 1870 par le décret Crémieux et acceptèrent avec enthousiasme de se voir " colonisés » par le Consistoire israélite de France. Une telle assimilation globale ne fut jamais proposée' aux Arabo-Musulmans pour qui elle aurait signifiée un abandon de leur identité musulmane. Ce nouveau statut des Juifs eut, entre autres, des conséquences éducatives et donc linguistiques.

Notre propos ici est de considérer la littérature judéo-maghrébine née de l'acculturation à la France sous l'angle linguistique. La question posée est de savoir si le français dans lequel ces ouvres sont écrites est perméable ou non aux langues juives maghrébines antérieures à la colonisation. Dans l'affirmative, les modalités de cette cohabitation seront à étudier afin de déterminer s'il y a persistance du modèle traditionnel de coexistence pacifique des langues.

La production littéraire de ce courant, quoique minoritaire, compte quelque 400 références. Elle est répartie sur une période d'un siècle mais marquée par une fracture. Avec les guerres d'indépendance nationale et la fin de la colonisation, les 
communautés juives des trois pays đu Maghreb émigrent vers la France mais aussi vers d'autres pays francophones, le Québec, la Belgique, et vers Israèl. Sur 120 écrivains dénombrés (Dugas, 1992), un seul continuera à vivre et à produire dans l'Algérie indépendante.

Notre étude devra donc considérer la question des langues dans cette littérature avant et après cette ligne de fracture que l'on fixe habituellement à 1962. Elle sera donc nécessairement diachronique.

\section{Co-présence pacifique}

De 1896, date à laquelle est publié le premier roman écrit conjointement par un Juif d'Algérie et un Français de la Colonie à 1962 où les trois pays du Maghreb, désormais indépendants, se vident massivement de leur population juive, les textes littéraires qui pratiquent la polyglossie juxtaposent les langues. Le français est émaillé d'emprunts à d'autres langues. Le plurilinguisme se donne à voir au niveau typographique : italiques, guillemets, notes en bas de page. Les citations qui suivent sont extraites du roman de Blanche Bendahan, Mazaltob qui fournit un exemple rare de quadriglossie : français, arabe, judéo-espagnol, hébreu.

- arabe : " balek sidi » (p. 95).

- judéo-espagnol : " Escuchis, senor soldado » (p. 115).

- hébreu : les « Abélim » (p. 105).

Alors que les occurrences dans les deux premières langues ajoutent au texte français une incontestable couleur locale, les incursions de l'hébreu se situent à un autre niveau. Ce sont des références savantes, religieuses pour la plupart :

"Sépharadim signifie en hébreu : espagnols. " (p. 15)

"Nédaba (de l'hébreu). Sorte de contribution volontaire à laquelle se soumettent les Juifs en faveur de leur race. " ( $p$. 25)

" Au kiddouch - ou bénédiction du vin... 》 (p. 158) 
La différence de nature entre, d'une part, les emprunts aux langues vernaculaires et, d'autre part, les emprunts hébraïques se mesure également à la longueur de l'énoncé enchâssé : membre de phrase pour les premières, mots isolés pour la seconde; ce qui apparaît nettement dans les exemples ci-dessus.

La traduction intervient sous diverses formes. Elle peut suivre immédiatement l'emprunt : « La Judéria - ou quartier juif ignore les saisons. " (p. 12). ou même le précéder : "Veille de samedi ou « noche sabbat » (p. 33), emprunt et traduction étant liés par un " ou " explicatif : la traduction-calque d'expressions figées comme dans la réplique suivante : $«-$ Parbleu, je comprends : Petit tamis de soie neuf $"$ nécessite l'ajout d'une longue note en bas de page bien qu'elle soit prise dans un tissu explicatif aussi bien sémantique (verbe comprendre) que syntaxique (ponctuation par les deux points). La note comporte le texte de départ, une traduction du type équivalence sémantico-pragmatique et une explication : « Traduction littérale de : Sédacito nuevo. Cette phrase veut dire: Tout nouveau, tout beau. (Quand le tamis est neuf, la farine passe mieux que d'habitude.) » (p. 16)

La situation linguistique est exposée dans le roman pour ce qui est de la langue juive de fusion, le judéo-espagnol :

Notre langue à nous, Séphardim, est l'espagnol ou ladino (de latino ou latin). Ce ladino - ce " haquétia " du bas peuple tétouanais -, qui se parle particulièrement sur la côte méditerranéenne, de Tanger jusqu'à Salonique; ce ladino qui est de l'espagnol de $1492 \ldots$ (p. 113) $^{1}$

Elle est exposée également pour la concurrence entre les langues coloniales en présence dans le Nord du Maroc, à savoir l'espagnol et le français. Les raisons du choix du français, malgré une situation de départ défavorable pour des « descendants de ces Juifs au parler

1. Pour une description scientifique de ces langues consulter $\mathrm{H}$. V. Séphiha $(1979,1986)$. 
archaïque de l'ancienne Castille " sont exposées en note. L'auteur cite en les traduisant quelques courts passages de l'ouvrage de Manuel L. Ortega " Los Hebreos en Marruecos » où les rapports de force entre l'Espagne et la France par zone d'influence linguistique sont évoqués. Le texte du roman est, quant à lui, un paradis où coexistent aussi bien la fidélité à l'héritage linguistique traditionnel - hébreu biblique et talmudique - qu'à celui de l'exil espagnol. Le réalisme dans l'utilisation de la langue de l'exil nord-africaine s'allie à la fierté d'avoir acquis par l'étude " la langue de Voltaire » (p. 10).

En choisissant de situer son roman dans la communauté juive de Tétouan qui fut au début du siècle un véritable carrefour linguistique où quatre langues : hébreu, arabe, espagnol et français se côtoyaient (S. Leibovici, Chronique des Juifs de Tétouan, pp. 180-189), Blanche Bendahan nous a laissé un texte littéraire en français dans lequel se mirent trois autres langues. La langue de la narration est le français, la langue de la religion est l'hébreu, la langue de la tradition est le judéo-espagnol, la langue du vécu en dehors de la sphère communautaire est l'arabe.

D'autres écrivains ont, dans le même esprit et avec un éventail de langues plus restreint, introduit dans la trame du français de nombreux emprunts à l'arabe ou au judéo-arabe et $/$ ou à l'hébreu.

Dans le recueil de contes de RYVEL (1930), de facture classique, le purisme du français s'allie aux références savantes en hébreu, les emprunts à l'arabe demeurant très rares.

Contrairement aux deux écrivains précédents chez lesquels les emprunts à l'hébreu étaient précis, chez les deux suivants les termes hébraYques sont pour la plupart erronés. La tradition et la superstition ont pris le pas sur la religion mais ceci n'affecte en rien la coexistence pacifique entre les trois langues : français, arabe, hébreu. 
Le roman d'Élissa Rhaīs, Les Juifs ou la fille d'Éléazar est truffé d'emprunts. Cas unique : certains termes rituels apparaissent en caractères hébraïques au sein du texte français, avec une orthographe fautive, comme $ר$ ל au lieu de $7 V$ (p. 79) et ' ${ }^{\prime} T \mathrm{~W}$ (p. 185). Mais ce peut être une erreur de typographie. Translittérés, ils reflètent la prononciation locale influencée par l'arabe : " mecvé » pour mikvé (p. 184), " choumir " pour chomer (p. 37). Nombre de mots arabes translittérés ne sont pas en italique. Considérés comme usuels, ils se fondent dans le français, ainsi " derbouka " (p. 261), " hammam », « khelkhal » (p. 147). Par contre, les phrases en arabe sont traduites : "Ou ma ikoun ghir khatrek! Et il n'y a que ton cour qu'on satisfera! " (p. 103). Traduction libre et romantique pour : Et ce ne sera que conformément à ta volonté !

Ce roman accueille en outre deux autres idiomes, dont l'un est le français zozoté sous influence de l'arabe. Notons, dans l'exemple ci-dessous, le mot " famille » arabisé par ajout de la désinence arabe du féminin pluriel :

moussieur Edmoun, c'est toi qui soisis... Au zardin Marengo, zeudi proçaine, dix heures...Y en aura cinq ou six familiates... (p. 103)

L'autre langue qui fait ici une apparition timide tant originale qu'inattendue est le judéo-alsacien. L'un des personnages, quittant son Alger natal pour poursuivre des études rabbiniques à Paris, y rencontre pour la première fois des juifs ashkenazes, originaires d'Alsace. Deux mots dont le sens se déduit du contexte : " shul 》 et « matses » sont insérés avec note signifiant que " le mot semble appartenir au patois alsacien $»$. (p. 74 et p. 80). Cet exemple est éloquent. La présence de mots d'une langue dont l'auteur n'ignorait peut-être pas l'existence mais qu'elle ne pratiquait 
certainement pas, illustre bien cette capacité d'accueil de langues qui se fonde sur une longue tradition ${ }^{2}$.

Dans le roman d'Irma Ychou, La famille Bensaïd, la part de l'hébreu et celle du judéo-arabe sont insignifiantes par rapport au grand nombre d'emprunts à l'arabe. L'hébreu translittéré reproduit la prononciation locale : " Youm Kippour » pour Yom Kippour (p. 92). La profession de foi juive est tronquée de même que sa traduction. L'explication donnée en note : « Ceci est le début de la prière des morts chez les Hébreux " est erronée mais notre auteur reproduit une erreur qui avait cours dans les milieux populaires.

Dès les premières pages, la question de la langue utilisée par cette famille juive du Sud algérien est évoquée : " Entre eux, ils parlaient indifféremment arabe ou français. » (p. 9)

De veine réaliste, le roman abonde en mots et phrases en arabe. La présence ou l'absence des guillemets et des italiques ne semblent pas répondre à une règle bien précise, la traduction de mots isolés est souvent absente quand le sens générique est facilement compréhensible. La traduction des phrases est donnée en incise ou en note. Elle est généralement exacte ${ }^{3}$.

L'interférence entre l'arabe et le français est telle qu'une réplique comme : " - $\grave{A}$ bas la religion des filles! grogna Bensaĩd 》 n'est en réalité qu'un calque de l'arabe : " n'al di nel-bnat ". L'écrivain qui s'est amusé à traduire mot à mot un juron populaire a opéré un transfert linguistique et culturel. Le lecteur

2. Il faut se souvenir que la rencontre entre Juifs alsaciens et Juifs algériens s'était faite après 1870 lorsque, à la suite de la perte de l'Alsace-Lorraine, de nombreux Juifs s'installèrent à Paris. Le Consistoire israélite de France délégua alors des rabbins alsaciens en Algérie.

3. Nous n'avons noté qu'un seul cas de phrase non traduite à la page 42. Il s'agit d'une injure à caractère scatologique. 
non-arabophone aura néanmoins saisi la force illocutoire de l'énoncé.

Le choix de ces trois romans n'est pas fortuit. Nous avons montré ailleurs à quèl point les romancières avaient facilement innové, en optant pour un genre littéraire - le roman - qui ne plongeait pas dans les racines de la littérature traditionnelle locale et en adoptant une attitude critique envers les sources religieuses ${ }^{4}$. Plus enthousiastes que les hommes à s'émanciper, elles étaient plus aptes ả renoncer à ces langues locales. Or, elles ne l'ont pas fait.

Le métissage des langues peut être interprété de deux manières. Il peut être un signe de continuité et reproduire le modèle que nous évoquions au début de cet article ou au contraire il s'inscrirait dans une rupture par rapport à la littérature traditionnelle. Auquel cas, il aurait pour but d'attirer le lecteur de la métropole sur une littérature de témoin, authentifiée par ces emprunts à des langues locales. Dans cette optique, il serait un moyen de fonder une littérature indigène exotique au sens noble du terme.

Dans cette littérature, le texte se présente comme une mosaïque ou un patchwork de langues où l'une complète l'autre, chaque langue venant exprimer ce qu'elle sait le mieux faire, dans une construction du sens, fortement ancré dans un territoire. La multiplicité des langues exprime l'identité plurielle d'un sujet qui vit ses diverses allégeances sur le mode de la complémentarité et de la richesse mutuelle. Il n'est pas ce " m'tourni ", ce converti, ce renégat qui doit changer d'identité. La co-présence des langues reflète la coexistence harmonieuse des composantes identitaires de leurs utilisateurs'.

4. Cet aspect a été développé dans une précédente contribution (Touitou-Benitah, 1994a).

5. Ce terme de « m'tourni » est composé à partir d'une racine française - tourner - prise entre un préfixe et un suffixe arabes. La nouvelle d'Isabelle Eberhardt qui porte ce titre peut 


\section{Rupture}

Cette Babel, qui se voulait et qui se croyait heureuse n'attendra pas l'exode massif des Juifs et les indépendances nationales pour disparaître. La colonisation réussira à imposer sa langue au détriment des langues locales, à la parer de l'auréole de la Civilisation, rejetant les cultures indigènes dans la déconsidération (Calvet, 1979, pp. 60-85). C'est du moins ainsi que la majorité des Juifs nord-africains, acculturés et en voie d'assimilation, "évolués ", l'avait comprise et vécue (Lévy-Mongelli, 1980). Dans les années 50 , le monolinguisme de règle se reflètera dans une littérature juive nord-africaine qui occultera toute langue autre que le français.

Deux écrivains dont le texte est absolument net de toute trace de langue locale fournissent une approche totalement différente, selon que leur acculturation est en voie d'accomplissement ou déjà accomplie.

Albert Memmi, qui, dans son premier roman la Statue de $s e l$, avait octroyé à sa langue maternelle - le judéo-arabe tunisien la désignation dédaigneuse de patois, écrit à ce propos :

Ma langue etait, en effet, en fusion, un infâme mélange d'expressions littéraires ou même précieuses, de tours traduits du patois, d'argot d'écolier et d'inventions verbales plus ou moins réussies. (p. 101, éd. 1966)

Mais le texte ne nous donne aucun exemple de ces « tours traduits du patois ». Dans deux de ses romans ultérieurs, le Scorpion et le Désert, des documents en caractères arabes et hébraïques sont insérés, la langue du texte littéraire demeurant exclusivement le

être lue comme l'illustration de la coexistence pacifique du français et de l'arabe. Une telle lecture humaniste ne prend pas à son compte les soubassements idéologiques et politiques assimilationnistes. 
français. Ces reproductions d'images calligraphiques sacrées (le Scorpion, pp. 127, 232), (le Désert, p. 174) font de l'arabe et de l'hébreu des icônes à admirer à distance. Les patois, langues hybrides entre l'arabe et l'hébreu, n'ayant pas droit de cité dans l'écrit.

Nombre d'écrivains totalement acculturés et exclusivement francophones n'ont donné aucune place aux langues maternelles de leurs parents et de leurs grands-parents qu'ils ignoraient ou dont ils ne connaissaient au mieux que quelques bribes. A la limite, il ne s'agit plus là de littérature jứéo-maghrébine.

À la morgue affichée par Albert Memmi, il faut opposer l'attitude valorisante de Jean Daniel. Dans un premier roman également, l'Erreur, il évoque cette langue de la mère - qui n'était pas sa langue maternelle - empreinte de spiritualité et de poésie :

[...] elle [ma mère] commençait, implorante et digne, les admirables mélopées judéo-arabes qui constituaient sa prière. (p. 96, éd. 1977)

La langue pré-coloniale n'est plus pour Jean Daniel une concurrente à neutraliser. Reléguée au rang d'une archive familiale, elle a droit aux honneurs de l'émotion. Le monolinguisme n'est pas contraint, il est un état de fait.

La rupture est fonction du degré d'acculturation des écrivains. Cette rupture est également datée. C'est dans les années 50 , au moment où la coexistence pacifique n'est plus possible en Afrique du Nord, que la polyglossie s'efface. Les dès sont jetés et les jeux sont faits pour la quasi totalité des écrivains. Le français sera le seul horizon culturel et linguistique.

\section{Co-présence recréée et créatrice}

Pour ceux d'entre les écrivains qui quittèrent l'Afrique du Nord à l'âge adulte et conservèrent une connaissance - parfois passive ou lacunaire, parfois excellente - de ces langues, la présence 
d'emprunts aux autres langues dans le texte littéraire se négocie à l'aune du souvenir.

Le festin des langues est tout d'abord festin de la langue et du palais quand le souvenir des délices culinaires que la mère préparait avec tant d'amour est évoqué. Des mots comme : la " dfina ", la « meguina ", les " asbanes 》, les " mekerkechettes", les « korsates », les « sefendjs » (Camille El-Baz, Sarah, pp. 38-39, 163) qui désignent divers mets et patisseries traditionnels ont gardé toute leur saveur.

La nostalgie de la langue est une fête où règnent le sourire et le rire. La recréation de la Babel linguistique des origines est aussi le dernier effort pour ne pas perdre définitivement quelque chose qu'on ne peut pas ou qu'on ne veut plus transmettre.

Katia Rubinstein s'applique dans un premier ouvrage, Mémoire illettrée d'une fillette d'Afrique du Nord à l'époque coloniale, à restituer avec une cocasserie qui frise la vulgarité , le parler des quartiers populeux de Tunis où coexistaient Juifs, Siciliens, Arabes. Les emprunts à l'arabe, à l'italien et les calques de l'arabe sont très nombreux. Aucun particularisme typographique ne les isole d'un français à l'orthographe souvent phonétique et à la syntaxe de l'oral. Les traductions sont intégrées au flux de la parole :

- allez, va! ti va b'rass ommek ma fille, sur là tête de ta mère!; la vérité, c'est ton tour, juste ou pas? (p. 73)

- ...basta! basta! mal educato! senza vergogna! mascalzone! chuidi il pick-up! subito! subito! assez! assez! mal élevé! sans vergogne! mal fagoté! eteins le pick-up! vite! vite! (p. 145).

Ami Bouganim dans Récits du Mellah restitue la situation linguistique vécue dans l'enfance au Maroc avec un humour qui ne peut laisser indifférent :

Le matin : le français; l'après-midi : l'arabe; le soir : l'hébreu. Avec des parrains gaulois, des tuteurs arabes et des ancêtres 
hébreux, j'éprouvais après ma déconfiture messianique, un grand mal à me redéfinir une vocation. Mais là aussi, je compris très vite la combine et me mis à jouer sur trois claviers alphabétiques à la fois : je mentais en français, j'insultais en arabe, je priais en hébreu... (p. 212).

Les emprunts à l'arabe et à l'hébreu sont traduits, au début en note en bas de page. Mais assez rapidement, ils sont intégrés au texte et la traduction suit généralement. La traduction est prétexte à jeu comme dans cet épisode où un rabbin défroqué, devenu professeur de philosophie, attise la curiosité de ses ex-ouailles à qui il faut expliquer ce qu'est la philosophie :

Harcelé, le Président se résigna à bafouiller la définition du Larousse : " Dkhmima elmsdra dla'kel ... " ou en français : " Étude rationnelle de la pensée humaine du double point de vue de l'action et de la connaissance. » (p. 53)

Bouganim n'a traduit que la première partie de la définition du Larousse. Sa pratique du judéo-arabe ne lui permettant pas d'exprimer des notions abstraites.

Nous avons relevé deux cas de non-traduction d'expressions figées de même nature, l'une en jưéo-arabe (p. 53), l'autre en araméen (p. 146) qui précèdent l'énonciation d'événements traumatiques ou dramatiques et en neutralisent l'effet'. Bouganim les utilise, bien entendu, ironiquement.

La nostalgie de la langue chez les écrivains d'origine algérienne est celle du français populaire « pied-noir », le pataouète. Toute une ceuvre peut être marquée par la recherche de la langue en voie d'extinction. Deux écrivains sont à un degré sans précédent des orfèvres de cette langue : Roland Bacri et Albert Bensoussan. Pour respecter le cadre de cette étude, nous avons choisi de nous

6. Nous avons appelé ces expressions " énoncés conversifs 》 (Touitou-Benitah, 1994b). 
concentrer sur l'œuvre de Bensoussan dans laquelle la rencontre des langues et des cultures est plus accusée?

Albert Bensoussan, traducteur et fils d'interprète militaire, poursuit depuis trente ans une cuvre où les jeux de langue font foi d'une créativité lexicale débordante. L'arabe investit le français. $E$. Schousboe (1991, pp. 113-118) ne traite du plurilinguisme de Bensoussan que sous l'angle de la communauté de langue entre Juifs et Arabes. Elle insiste sur les regrets de Bensoussan d'avoir été coupé par l'éducation française du judéo-arabe de ses ascendants mais ne met pas en évidence la virtuosité avec laquelle il a su, à partir de bribes, reconstituer un univers linguistique où le sépharadisme et l'hébraïsme occupent au fur et à mesure des publications de plus en plus de place. Les romans de Bensoussan sont tous des romans de la langue. La fête verbale retrouve son éclat du passé avec des emprunts à l'arabe, à l'hébreu, à l'espagnol. D'un livre à l'autre, les emprunts perdent leurs italiques et se fondent en une langue qui célèbre, peut-être pour la dernière fois, une harmonie que la nostalgie a certainement embellie. « Ce bien le plus précieux, ce puits sans fond " (le Félipou, p. 9) qu'est la langue se prête à tout.

Alger, la ville natale tant chantée, devient Frimaldjézar, mot constitué par l'enchaînement de frime et du nom arabe d'Alger, el-Djézaïr. Séville est Isbilia, le nom arabe de la ville s'est substitué au nom français ou espagnol pour évoquer la nostalgie de l'âge d'or espagnol. Les récits de la Genèse sont prétexte à pastiche où l'ordre des deux épisodes, celui de la création et de Babel, est inversé, donnant à la langue la première place : " En ce temps-là on n'avait pas encore fait le partage des langues et la séparation des eaux. "

7. Notons toutefois que Bacri a publié, outre ses recueils de poèmes et ses romans, un dictionnaire du français pataouète commenté de manière fantaisiste et un introduction humoristique au parler « pied-noir » éditée dans le cadre d'une collection sur les parlers régionaux. 
(Frimaldjézar, p. 157) Séville remplace Jérusalem dans ce calque de l'hébreu des Psaumes (CXXXVII, 5):

Que ma droite se dessèche

Isbilia

si je t'oublie! (Visage de ton absence, p. 102),

Reproduisant la façon des poètes hébraiqques de l'Espagne médiévale, il intègre des passages de la liturgie ${ }^{8}$. Ainsi, la traduction des bénédictions du matin peut passer inaperçue dans ce passage (le Dernier devoir, p. 86) :

Surtout chez nous, à Alger, où chacun mettait son point d'honneur à proclamer plus haut que le voisin que l'Éternel rend la vue aux aveugles, que le Roi de l'univers délivre les captifs, que notre Dieu relève ceux qui sont abaissés et donne des vêtements à ceux qui sont nus. On le sait, on l'a dit, on l'a lu.

Parfois, la traduction de l'hébreu est plus apparente, voire déclarée : « [...] ton Benjamin - le fils de tes jours, comme dit l'hébreu » (le Dernier devoir, p. 63)'.

La traduction mot à mot est un jeu avoué : « Rosh Hodèche - la tête du mois - traduisais-je ingénument " (le Félipou, p. 38). Le calque et la substitution superposent une culture à une autre et font naître le sourire : " et Rabbi Chim'on Bar-Yohai n'était pas mon cousin " (le Dernier devoir, p. 25).

8. Le poète Moche Ibn Ezra avaìt au $\mathrm{XI}^{\natural}$ siècle procédé de même quand il fut obligé de quitter l'Andalousie pour le Nord de l'Espagne.

9. Il y a erreur de la part de Bensoussan. Benjamin veut dire en hébreu " fils de la droite ", " fils de la vieillesse " (Genèse, 35,18 ). 
Une critique de l'hypocrisie religieuse et sociale peut se cacher derrière une homophonie presque parfaite entre le mot hébreu désignant le Mur des Lamentations et le mot cocktail : « chacun [...] court s'incliner au kotel - ou dit-on cocktail? - bon chic bon genre " (le Dernier devoir, p. 28).

Le rapprochement entre le français et l'hébreu peut aller jusqu'à cette définition linguistique pour le moins inattendue : " Le tréma, fragile daguesh des te'amim, vole au moindre zéphyr beauceron " (le Marrane ou la confession d'un traitre, p. 57) .

L'arabe transparaît dans des calques comme : « Son ceil est sa balance " (le Félipou, p. 11). Cette traduction avec passage de la seconde à la troisième personne de "'einek mizanek ", pirouette généralement utilisée par les femmes qui transmettent les recettes de cuisine sans spécifier les quantités exactes. Cette expression, dans son imprécision, est ambivalente car tout en affirmant la maitrise de celle qui livre la recette, elle accuse l'inexpérience de la novice. Bensoussan ne pouvait que tirer parti d'une telle expression qu'il transpose, bien évidemment, dans un contexte tout différent. Quelques lignes plus bas, un néologisme comme " enchéché " est constitué par le mot arabe " chèch" ou " chéchia " qui désigne une calotte de laine, pris entre un préfixe et un suffixe français, sur le mode de " encapuchonné ".

Relevons pour terminer cet échantillonnage un jeu de mots digne du traducteur qu'est Bensoussan. Le quartier populeux d'Alger, Bab-el-oued est celui de son enfance où vivaient des communautés diverses, idéalisé par des expressions comme " Babel-oued " et "l'atour de Babel » et non pas le Bab-el-oued des années de guerre, bastion des Pieds-noirs (le Marrane ou la confession d'un traître, p. 64). Une fois encore la langue (Babel, le

10. Le daguesh est un signe qui équivaut au redoublement de la consonne tandis que les te'amim sont les signes de cantilation du texte biblique. 
partage des langues) et l'eau (oued, la séparation des eaux) sont liées comme sources de vie.

\section{Conclusion}

Le métissage des langues des écrivains judéo-maghrébins contemporains peut dérouter le lecteur non-initié à ces langues" ${ }^{\mathrm{Il}}$. Le désir de faire revivre une communauté, une culture dans son foisonnement linguistique est plus fort que le souci de lisibilité, pourtant primordial pour un écrivain. Le jeu des langues qui se voulait dans les années 20 et 30 volonté de plaire et volonté d'atteindre le plus grand nombre par un parfum d'exotisme devient aujourd'hui, pour certains, après la rupture des années 50 et 60 , l'expression d'un particularisme qui vise les frères de langue, dans la joie des retrouvailles, dans l'au-delà du traumatisme de l'exil. Joie que vient assombrir le sentiment profond qu'avec cette dette payée à la mémoire, sonne le glas de la coexistence de ces langues.

La plupart de nos auteurs (Bendahan, Ychou, Memmi, Bouganim) ont estimé nécessaire d'exposer la situation linguistique qui a été la leur. Bensoussan a déploré le fait de n'avoir pas été ce bilingue qu'il aurait voulu être. Ce judéo-arabe qu'il n'avait pas acquis en Algérie, il devra le conquérir de haute lutte en France. Le plurilinguisme rapporté a posteriori comme un bienfait, un enrichissement, a-t-il été vécu de la sorte?

Daniel Sibony (l'Entre-deva), ayant lui-même vécu cette polyglossie dans son enfance judéo-maghrébine, se souvient de ses zéros en rédaction car le professeur supportait mal ses mélanges " farcesques » entre le français et sa culture indigène. Contrairement à ses appréhensions, en France, son professeur appréciera cette façon originale d'écrire.

11. Signalons que Rolland Doukhan ajoute a la fin de son premier roman au titre hébraique Berechit (Denoèl, 1991) un lexique de termes hébraïques, arabes et yiddish. 
La colonisation et l'acculturation ont combattu la co-présence des langues qui n'est devenue festin qu'après l'exil avec la recréation de ces entre-deux-langues, entre-trois-langues. Pour Sibony, les récits de l'origine qui s'offrent en partage sont l'expression d'une identité tentant de recoller ses morceaux, de s'intégrer à elle-même en croyant s'intégrer à d'autres. C'est une " identité morcelée mais consistante " (p. 20) qui se reflète dans le miroir de textes où les langues sont semblables aux paillettes d'un kaléidoscope.

\section{Références primaires}

BACRI, Roland (1969). Le Roro. Paris, Denoèl. (1983). Trésor des racines patoouètes. Paris, Belin.

BENDAHAN, Blanche (1930). Mazaltob. Paris, Éditions du Tambourin.

BENSOUSSAN, Albert (1976). Frimaldjezar. Paris, Calmann-Lévy. (1988), Le Demier devoir. Paris, L'Harmattan.

(1990). Visage de ton absence. Paris, L'Harmattan.

(1991). Le Marrane ou la confession d'un traitre. Paris, L'Harmattan.

(1994). Le Félipou. Contes de la sixième heure. Paris, L'Harmattan.

BOUGANIM, Ami (1981). Récits du Mellah. Paris, Jean-Claude Lattès. 
DANIEL, Jean (1953). L'Erreur. Paris, Gallimard. Rééd. Livre de Poche (1977).

EBERHARDT, Isabelle (1986). Yasmina et autres nouvelles algériennes. Paris, Liana Lévi. Nouvelle " M'tourni » parue en 1905 dans l'Akhbar, puis éditée en 1920 dans Pages d'Islam, Fasquelle.

EL-BAZ, Camille (1971). Sarah ou mours et coutumes juives de Constantine (Algérie). Nice [publié à compte d'auteur].

MEMMI, Albert (1953). La Statue de sel. Paris, Correa. Rééd.Gallimard (1966).

(1969). Le Scorpion. Paris, Gallimard.

(1977). Le Désert. Paris, Gallimard.

RHAIS, Elissa (1921). Les Juifs ou la fille d'Eléazar. Paris, Plon.

RUBINSTEIN, Katia (1979). Mémoire illettrée d'une fillette d'Afrique du Nord à l'époque coloniale. Paris, Stock.

RYVEL [pseud. de Raphaël Lévy] (1930). L'Enfant de l'Oukala. Tunis. Éd. La Kahéna. Rééd. (1980) Paris, Jean-Claude Lattès.

YCHOU, Irma (1947). La Famille Bensaïd. Paris, Albin-Michel.

\section{Références secondaires}

BAR-ASHER, Moshe (1992). La Composante hébrä̈que du judéo-arabe algérien. Jérusalem, Éditions Magnes, Université hébraĩque.

CALVET, Louis-Jean (1979). Langue et colonialisme, petit traité de glottophagie. Paris, Payot. 
CHETRIT, Joseph (1980). " Niveaux, registres de langue et sociolectes dans les langues judéo-arabes d'Afrique du Nord ", les Relations entre Juifs et Musulmans en Afrique du Nord aux $X I X^{*}-X X^{*}$ siècles. Éd. J.L. Miège. Paris, Éditions du C.N.R.S., 1980, pp. 129-142.

(1994). ha-chira ha-'aravit - yehoudith chebikhtav bi-tsfon afrika. 'iyoumim poetiim, lechoniim we-tarboutiim [La poésie écrite judéo-arabe en Afrique du Nord. Étude poétique, linguistique et culturelle]. Jérusalem, Éditions Misgav Yerushalayim, Institut de recherche sur les communautés sépharađes et orientales.

DUGAS, Guy (1990). La littérature judéo-maghrébine d'expression française : Entre Djéha et Cagayoux. Paris, L'Harmattan.

(1992). Bibliographie critique de la littérature judéo-maghrébine d'expression française. Paris, L'Harmattan.

LEIBOVICI, Sarah (1984). Chronique des Juifs de Tétouan (1860-1896). Paris, Maisonneuve et Larose.

LEVY-MONGELLI, Danielle (1980). " Un cas particulier d'aliénation culturelle : les Juifs d'Afrique du Nord dans l'aventure coloniale française ». Juifs du Maroc. Identité et dialogue. Grenoble, La Pensée sauvage.

SHOUSBOE, Élisabeth (1991). Albert Bensoussan. Paris, L'Harmattan.

SEPHIHA, Haim Vidal (1979). Le Ladino (judéo-espagnol calque) : structure et évolution d'une langue liturgique. Paris, Association Vidas Largas.

(1986). Le Judéo-espagnol. Paris, Éd. Entente.

SIBONY, Daniel (1991). Entre-Dezx. L'origine en partage. Paris, Seuil. 
TEDGHI, Joseph (1995). Les Interférences de l'hébreu dans les langues juives. Paris, Centre de documentation et de recherche, Études hébraïques et juives modernes et contemporaines. Institut national des langues et civilisations orientales.

TOUITOU-BENITAH, Colette (1994a). « Transgression de la Loi, transgression du genre? Les romancières juives d'Afrique du Nord ". Créativité et imaginaire des femmes. Le genre et la loi II. Bordeaux, Éditions de la Maison des Sciences de l'Homme d'Aquitaine, pp. 139-148.

(1994b). " Les Énoncés conversifs », Interface IX.1 (1994), VLEKHO, Bruxelles, pp. 29-40.

RÉSUMÉ : De la co-présence pacifique à la co-présence créatrice : le kaléidoscope des langues dans la littérature judéo-maghrébine - Considérant la littérature judéo-maghrébine d'expression française depuis sa naissance en 1896 et jusqu'à nos jours sous l'angle de la co-présence de langues différentes dans le texte, trois périodes distinctes sont relevées. La première - de 1896 au début des années 50 - et la troisième - de 1962 à nos jours sont marquées par cette co-présence. Mais cette co-présence a un caractère différent au cours de ces deux périodes. De la co-présence pacifique où le texte français était émaillé de mots et expressions en langues juives - judéo-arabe et judéo-espagnol -, on passe à une co-présence créatrice où les langues se fécondent l'une l'autre. Tous les jeux sont permis pour restituer ces langues juives qui se perdent et que l'on ne peut plus transmettre. La période intermédiaire au cours des années 50 constitue une rupture puisque des textes monolingues attestent du triomphe de la colonisation linguistique.

ABSTRACT : Foreign words in the French Literature of the Jewish Maghreb : from Pacific to Creative Patterns of Co-occurrence- The French literature of the Jewish Maghreb from 1896 onwards records the presence of different foreign words in the original text. Two patterns of co-occurrence of langages are 
described defining two different periods : a first period from 1896 to the beginning of the fifties and a second period from 1962 to the present. During the earlier period, the foreign words taken from Judeo-Arabic and/or Judeo-Spanish are included in the French text without reciprocal influence between them. This kind of co-occurrence is termed the "pacific co-occurrence." On the contrary, the later period is characterized by interaction between the different langages in order to bring back to life Jewish langages which were not handed down anymore from one generation to the other and got lost. This pattern is termed the "creative co-occurrence." A middle period, around the fifties, is marked by a break since only monolingual texts were written attesting to the triumph of the French colonial policy.

Colette Touitou-Benitah : Translation department, Bar-Ilan University, 52900 Ramat Gan, Israël. 